\title{
Investigating the fission process at high excitation energies through proton induced reactions on ${ }^{181} \mathrm{Ta}$
}

\author{
Y. Ayyad ${ }^{1}$, J. Benlliure ${ }^{1}$, E. Casarejos ${ }^{1}$, H. Álvarez Pol ${ }^{1}$, A. Bacquias ${ }^{3}$, A. Boudard ${ }^{3}$, \\ T. Enqvist ${ }^{2}$, V. Föhr ${ }^{2}$, A. Kelic ${ }^{2}$, K. Kezzar ${ }^{3}$, S. Leray ${ }^{3}$, C. Paradela ${ }^{1}$, D. Pérez-Loureiro ${ }^{1}$, \\ R. Pleskac ${ }^{2}$, and D. Tarrío ${ }^{1}$ \\ 1 University of Santiago de Compostela, 15754 Santiago de Compostela, Spain \\ 2 Helmholtzzentrum für Schwerionenforschung, Planckstrasse 1, 64941 Darmstadt, Germany \\ 3 DSMIRFU/CEA, 91191 Gif-sur-Ivette, France
}

\begin{abstract}
In this work we have investigated total fission cross section of ${ }^{181} \mathrm{Ta}+{ }^{1} \mathrm{H}$ at FRS (FRagment Separator -GSI) at 1, 0.8, 0.5 and $0.3 \mathrm{GeV}$ with an specific setup, providing high accuracy measurements of the cross section values. We compare the results obtained in this experiment, with several calculations performed with the intra-nuclear cascade model (INCL v4.1) coupled to de-excitation code (ABLAv3p), according to two different models describing fission process at high-excitation energies: statistical model of Bohr and Wheeler and the dynamical description of the fission process. The comparison with data of previous experiments is also discussed in order to address the existing discrepancies with this new results.
\end{abstract}

\section{Introduction}

Fission at medium and high energies provide valuable information of the dynamics of the fission process [1]. It is also relevant in a wide range of applications, such as the construction of spallation neutron sources for Accelerator-driven systems. Despite the clear interest of these processes and applications, the available experimental information in this energy domain, and in particular for nuclei with low fissility, is scarce. Moreover, state-of-the-art models and data-based systematics show discrepancies with the available data.

One of the most demanding applications of this type of data is related to the spallation targets used for neutron sources, or accelerator-driven-systems. The fission reactions produced in the spallation blankets of those systems have a direct impact on their radioactive inventory. Additionally fission will contribute to the production of gases, namely $\mathrm{Kr}$, and therefore, has a direct impact in the structural properties of the whole target assembly. In this context, it was soon recognized the importance of measuring key reactions that would provide direct information on the fission residues of interest. These data, measured with enough accuracy, should constitute a data base to benchmark the existing reaction models. One of these reactions was the fission induced by high energy protons on $\mathrm{W}$ or Ta, candidates to be used as spallation targets. It exists few experimental data, with discrepancies, about the protoninduced fission at medium and high energies of Ta.

In this work we present new experimental data of proton-induced fission on ${ }^{181} \mathrm{Ta}$. We have measured the total fission cross-section, at different energies: 300, 500, 800 and $1000 \mathrm{AMeV}$. By using the inverse kinematic technique and a dedicated experimental setup, we measured the data with high accuracy. Our results allows for benchmarking the sate-of-the-art models, and provide data for systematic descriptions.

This is an Open Access article distributed under the terms of the Creative Commons Attribution-Noncommercial License 3.0, which permits unrestricted use, distribution, and reproduction in any noncommercial medium, provided the original work is properly cited. 


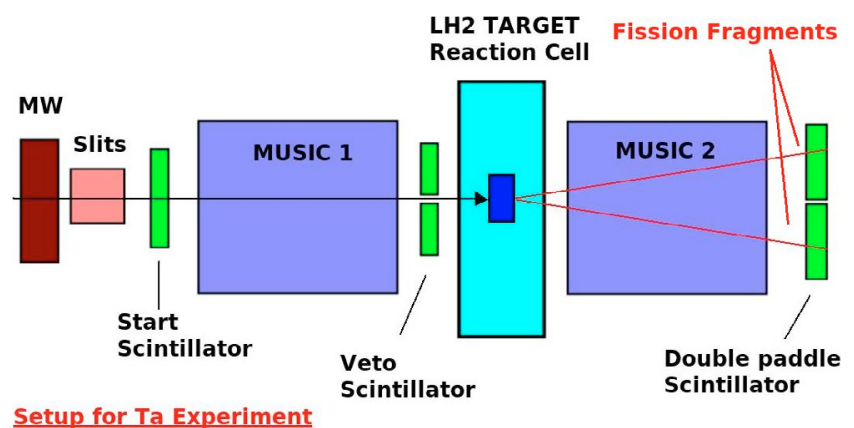

Fig. 1. Experimental Setup. Inverse kinematics.

\section{Experiment}

The experiment was performed at the Helmholtzzentrum für Schwerionenforschung, Darmstadt, Germany (GSI), using inverse kinematics. A ${ }^{181}$ Ta beam accelerated up 1000, 800, 500 and $300 \mathrm{AMeV}$ was used in different runs. The beam impinged into a liquid hydrogen target $\left(85 \mathrm{mg} / \mathrm{cm}^{2}\right)$. The protoninduced reaction residues, which flew downstream, were analyzed with the detector setup sketched in fig. 1 .

In this experiment we focused on a high-accuracy measurement of the fission channel, which was expected to be about $1 \%$ of the total reaction cross-section. This challenging study required a capable and robust experimental setup. The Multi-wire chamber (MW) and slits, were used to guide the beam. The first scintillator (start) was used to account for the beam dose. The beam-halo particles and missaligned trajectories were rejected with the veto scintillator ( $15 \mathrm{~mm}$ bore) placed just before the target entrance. Two Multi-Sample Ionization Chambers (MUSIC), measured the energy loss of the particles passing through them. They were used, as explained below, to identify reactions of ${ }^{181} \mathrm{Ta}$ produced only in the hydrogen target, and not in any other layer of matter placed along the beam line.

Two scintillator paddles were mounted one on top of the other, downstream the second MUSIC. The coincidence of the signals from both, provided the fission trigger. The geometry of this trigger fits with the spatial distribution of the fission events. Other channels that maybe included in this trigger (background) were either eliminated or evaluated as described below.

\section{Data analysis}

Our analysis proceeds carefully to isolate the fission events measured with our setup, for each beam energy. In a first step we used the information from the two MUSIC chambers to identify the fission reactions at the hydrogen target. In a second step, the background of the previous selection was either rejected or evaluated, using the two scintillator paddles also used as trigger. These data were normalized to the beam dose and target thickness to provide the cross section. The contribution to the fission cross section of the target cell itself was also corrected.

Fig. 2 shows a scatter plot of the energy losses in the two MUSICs before and after the target (both in arbitrary units). The particles lying in the diagonal of the plot, correspond to ions which keep their atomic number when passing through the target, these are nuclei lighter than the primary beam that have been produced in nuclear reactions induced by ${ }^{181}$ Ta projectiles in any layer of matter placed upstream the hydrogen target. The dominant ${ }^{181} \mathrm{Ta}$ spot is clearly visible on the top. The reaction products of ${ }^{181} \mathrm{Ta}$ with hydrogen lay along the vertical line, just below the big ${ }^{181} \mathrm{Ta}$ spot. Heavy evaporation residues, immediately below of ${ }^{181} \mathrm{Ta}$, are the most populated group, as expected from the proton-induced spallation reactions with low fissility materials such as ${ }^{181} \mathrm{Ta}$. The fission products lay in the group of medium charges (MUSIC 2 channels around 2000, in the vertical axis).

Once we selected the events which are candidates, to be produced in ${ }^{181} \mathrm{Ta}$ fission reactions, we studied the background due to our trigger system. In fig. 3, we plot the energy loss measured in the 


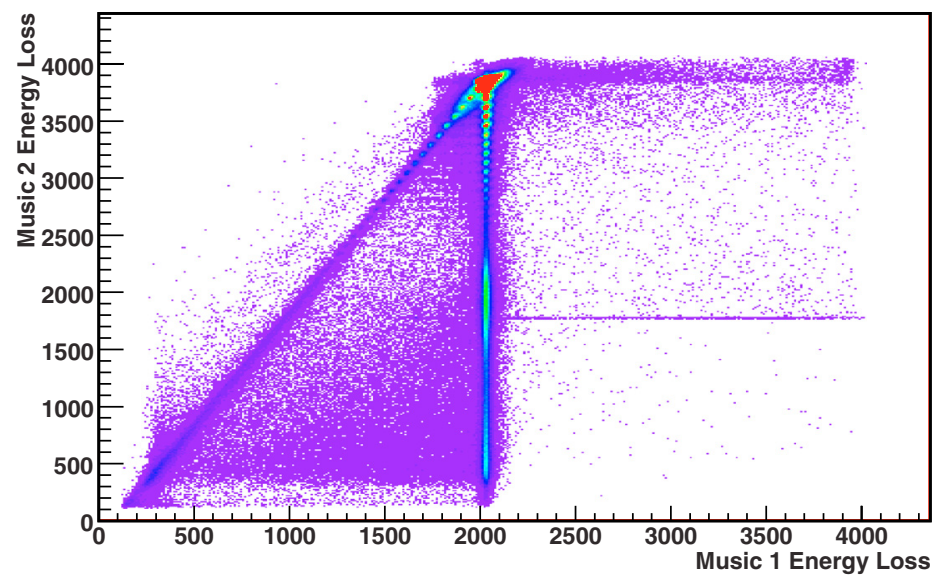

Fig. 2. MUSIC 1 vs MUSIC 2. Energy Loss. The diagonal line shows the reaction of the Ta beam with the detectors and other layers of matter. In the vertical line we recognize the Ta spot corresponding to the beam, and immediately below, the evaporation products. The fission region covers from 1000 to 2500 (a.u.) in the vertical axis.

two scintillator paddles (in arbitrary units) at $1000 \mathrm{AMeV}$ and $300 \mathrm{AMeV}$, using the condition stated before for the selected fission events in the scatter plot of the MUSICs. Due to the charge splitting of the fission process, the sum of the energy loss of the resulting residues is constant, and lies in a characteristic region in such a plot. The region of fission-events is clearly visible below the limit for the addition of fissioning-residues charges. And well separated from other channels much more populated. We also compare the identification of the fission events at $1000 \mathrm{AMeV}$ and $300 \mathrm{AMeV}$. The low fission cross sections at lower energies, seriously difficult the identification of the fission events because of the higher cross sections of the predominating reaction channels at those energies. However, the experimental setup presented here, resolve the fission fragments from other reaction channels, separating the fission events from the background with an additional effort in the data analysis.

With this double selection procedure we have clearly isolated the fission channel contribution. In order to provide an accurate measurement of the cross section, we also evaluated the background which remains in the region of fission, underneath the fission-spot. To evaluate the background, we sliced the fission region. Each slice was projected into a rotated reference system, which enhanced the profile of the contributions coming from background and fission. The fits to each of the two contributions defined the correction for the background suppression. At the same time we could evaluate the systematic uncertainty due to this correction, found consistently below 9\%. The contribution of the target foils (namely Ti) which surrounded the liquid-hydrogen, was measured using an hydrogen-empty target run, and using the same analysis procedure, for each energy. This contribution amounts up to $30 \%$, with an accuracy of $6 \%$. To obtain the cross-section of the fission channel, we used the counting of the selected fission events, corrected for background and target-foils, and normalized to the beam dose (uncertainty of $2 \%$ ), and the target thickness (uncertainty of $4 \%$ ). The results are shown in table 1.

\section{Results and discussion}

The results of our measurements of the total fission cross-section of ${ }^{181} \mathrm{Ta}+{ }^{1} \mathrm{H}$ at different energies, are summarized in the table 1 . In fig. 4 we plot the available data for proton-induced fission on ${ }^{181} \mathrm{Ta}$, expanding from some $150 \mathrm{MeV}$ to $1000 \mathrm{MeV}$. We also compare the results given by state-of-the-art models and the systematics of Prokofiev [4].

The comparison of our data with the previous results, reveals a good agreement at high energies, from 700 up to $1000 \mathrm{AMeV}$. Hence, this work confirms the previously measured data with lower uncertainties. However, the situation remains unclear at the lower energies. In general, our results, covering a wide range in energy, are more smooth than the those from Yurevich et al. [9], showing an 

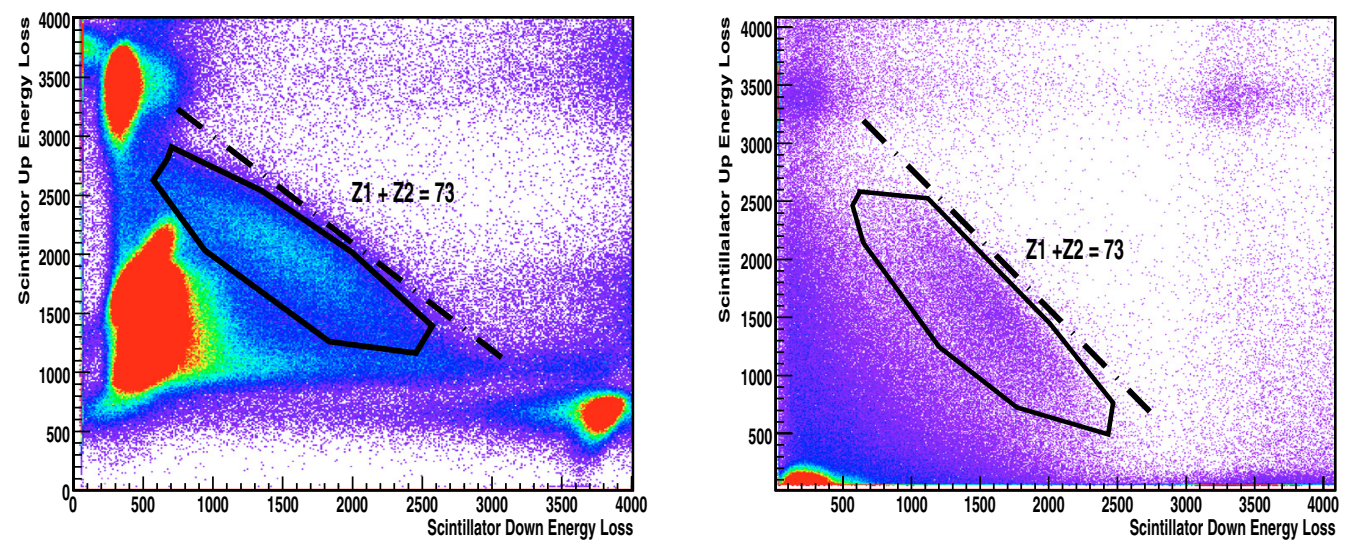

Fig. 3. Energy loss of the fission fragments in the double scintillator (Left panel $1000 \mathrm{AMeV}$ - Right panel $300 \mathrm{AMeV}$ ). Dashed line corresponds to $Z_{1}+Z_{2}=73$. Fission events are selected in the region below this line. For $300 \mathrm{AMeV}$ the identification of fission events becomes more difficult because of the small fission cross section.

Table 1. Total fission cross section ${ }^{181} \mathrm{Ta}+{ }^{1} \mathrm{H}$.

\begin{tabular}{ccc}
\hline Beam Energy & $\sigma(\mathrm{mb})$ & $\epsilon(\sigma)(\mathrm{mb})$ \\
\hline $1000 \mathrm{AMeV}$ & 18.49 & 3.93 \\
$800 \mathrm{AMeV}$ & 11.40 & 1.85 \\
$500 \mathrm{AMeV}$ & 5.14 & 1.36 \\
$300 \mathrm{AMeV}$ & 2.79 & 1.39
\end{tabular}

unclear step-like evolution with energy. It is important to remark the difference between the existing values and our data at those energies. The higher value of the fission cross section corresponding to other works shown here, could be due to the contamination of predominant reactions at lower energies that increase the final value of the fission cross section.

Fig. 4 shows a comparison between the existing measurements and fission cross sections systematics established by Prokofiev. We found a good agreement at high energies, where the prediction follows the trend of the experimental data. However at lower energies, the systematics over-predict the measured cross sections in our work. The systematic evaluation (dashed line) diverges up to $50 \%$ from our experimental data (solid circle).

The comparison of our data with model calculations, opens a discussion concerning the dynamics of the fission at high energies based on the results obtained in this work. In reactions induced on the ${ }^{181} \mathrm{Ta}$, the spallation process produces highly excited residues with high fission barriers. Under these conditions, the fission cross section represents a good observable of the fission dynamics taking into account the competition between fission and neutron evaporation in the excited residue. In this context the fission probability is related to the time the nucleus needs to fission.

We have used two codes for describing this reaction. These codes are based in a two-step scheme reaction. A first intra-nuclear cascade describes the collision; a de-excitation model describes the final residue distributions, and processes therein. A model coupling which has shown to provide realistic results is that of the INCL-v4.1 [2] cascade, and the ABLA-v3p [3] de-excitation to describe the reaction mechanisms of particle emission and fission. The statistical description of fission probability (BohrWheeler) is the most common picture for describing fission. This is a transition-state model based in a direct 'static' description of the fission process. In this model the fission probability depends on the available phase-space or level density above the fission barrier. However, it has been demonstrated that at higher excitation energies, a clear suppression of the fission channel appears when compared with the predictions of the statistical model [5]. This suppression is caused by the interaction of the internal degrees of freedom of the nucleons with the collective degrees of freedom concerning the movement of the nucleons [6]. Dissipation $(\beta)$ is quantified as energy exchange on this interaction, and leads to a 


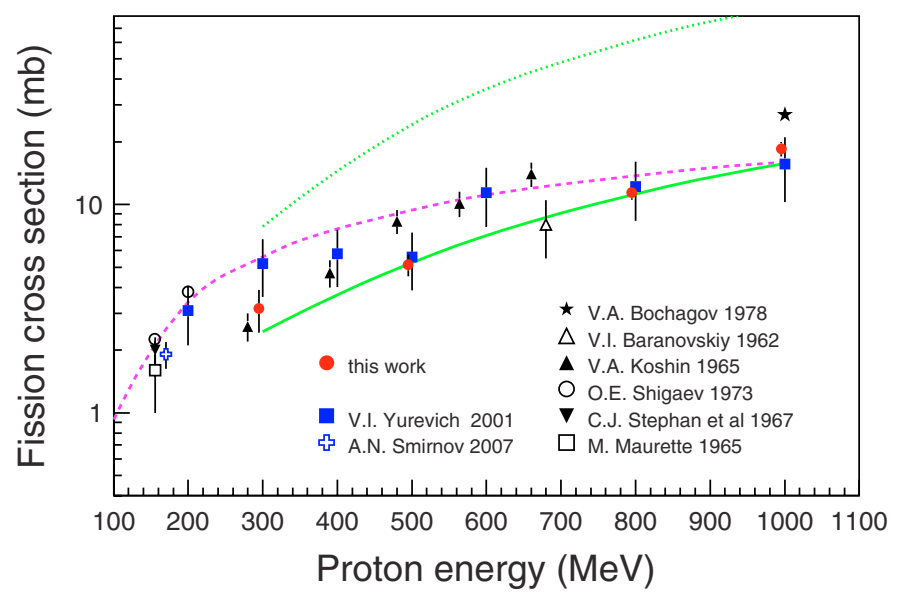

Fig. 4. Total fission cross sections for ${ }^{181} \mathrm{Ta}+{ }^{1} \mathrm{H}$. Dotted line: Statistical Model - Solid line: Dynamical model Dashed line: Prokofiev systematics - Solid circle: This work - Solid Square [9] - Cross [10] - Solid star [11] Open triangle [12] - Solid triangle [13] - Open circle [14] - Inverted Solid triangle [15] - Open Square [16].

delay of the fission channel due to the nucleus needs more to time to undergo fission, and the neutron evaporation competition could cool the nucleus down [7]. Hence, a dynamical description of the fission process including the dissipation, with possibility to emit particles in that process, has been found more suited to describe this regime [8]. These effects are clearly seen in fig. 4. The pure statistical approach overestimates those results far of the measured data (solid line). It is the dynamical description that provides realistic results (dashed line). The effect is more and more remarkable with increasing excitation energies.

\section{Conclusions}

In this experiment we have measured with high accuracy the total fission cross section of ${ }^{181}$ Ta induced by protons at different high energies: 300, 500, 800 and $1000 \mathrm{AMeV}$. We used the inverse kinematic method, and a specific detector setup to disnentangle the fission reaction channel from the rest of much more populated channels. The fission production and the corrections, as well as their uncertainties, were carefully evaluated.

Our results give new information in the discussion of the discrepancy among the various results. The existing systematics for the estimation of this reaction over-predict the result up to $50 \%$. This data also allows for benchmarking the models describing the fission process. Using state-of-the-art models, we showed that a simple statistical description largely over-predict the measured cross section. Only a dynamical description of the fission, considering the role of the viscosity of the nuclear matter, provides a realistic result.

\section{References}

1. B. Jurado et al. Phys. Rev. Lett. 93 (2004) 072501

2. J. Cugnon et al. Nucl. Phys. A 620 (1997) 475

3. J.-J. Gaimard, K-H. Schmidt, Nucl. Phys. A 531 (1991) 709

4. A.V. Prokofiev, Nucl. Instr. and Meth. A 463 (2001) 557

5. B. Jurado et al. Phys. Lett. B 553 (2003) 186

6. J. Benlliure et al. Nucl. Phys. A 700 (2002) 469

7. B. Jurado et al. Nucl. Phys. A 747 (2005) 14

8. J. Benlliure et al. Phys. Rev. C 74 (2006) 014609 
9. V.I. Yurevich, Fiz. Elementarnykh Chastic i Atomn.Yadra, Letters, Vol. 2, p. 49 (2005)

10. A.N. Smirnov et al. Int Conf on Nuc. Data f Science and Tech (2007)

11. V.A. Bochagov Soviet Journal of Nuclear Physics, Vol. 28, p. 291 (1978)

12. V.I. Baranovskiy Radiokhimiya, Vol. 4, p. 470 (1962)

13. V.A. Konshin Yadernaya Fizika, Vol. 2, p. 682 (1965)

14. O.E. Shigaev Khlopin Radiev. Inst., Leningrad Reports, No. 17 (1973)

15. C.J. Stephan Physical Review, Vol. 164, p. 1528 (1967)

16. M. Maurette Physics and Chemistry of Fission Conf., Salzburg 1965, Vol. 2, p. 307 (1965) 